\title{
The predictive effect of overexpressed miR-34a on good survival of cancer patients: a systematic review and meta-analysis
}

This article was published in the following Dove Press journal:

OncoTargets and Therapy

25 September 2015

Number of times this article has been viewed

Jian Wang'
Guorong Dan'
Jiqing Zhao'
Yu Ding'
Feng Ye'
Huiqin Sun'
Fan Jiang'
Jin Cheng'
Fahuan Yuan'
Zhongmin Zou'
'Institute of Toxicology, School
of Preventive Medicine, The
Third Military Medical University,
${ }^{2}$ Department of Nephrology,
Xinqiao Hospital, Shapingba District,
Chongqing, People's Republic of China

Correspondence: Zhongmin Zou Institute of Toxicology, School of Preventive Medicine, The Third Military Medical University, 30 Gaotanyan Street, Shapingba District, Chongqing 400038

People's Republic of China

Tel/fax +862368752290

Email zouzhmin@yahoo.com
Background: MicroRNA-34a (miR-34a) is a potential prognostic factor for survival in patients with several types of cancer according to previous clinical researches. We conducted a systematic review and meta-analysis to summarize the significance of increased miR-34a expression in the prognosis of patients' overall survival.

Materials and methods: The present systematic review and meta-analysis of 15 researches included 2,597 patients. Overexpression of miR-34a may predict good overall survival ([OS], $\mathrm{HR}=0.76,95 \%$ confidence interval: $0.55-1.06, P=0.105$ ), but the effect was not significant enough. Subgroup analysis results showed miR-34a was an ideal predictor for digestive system cancer (OS, HR $=0.50,95 \%$ confidence interval: $0.25-0.99, P=0.048)$. The predictive effects of elevated expression of miR-34a on the OS of untreated and treated patients were not of obvious differences.

Conclusion: This systematic review and meta-analysis showed that miR-34a has a predictive effect on overall survival of patients with digestive system cancer.

Keywords: meta-analysis, systematic review, miR-34a, cancer, prognosis

\section{Introduction}

MicroRNAs (miRNAs) are small single-stranded noncoding RNAs of $\sim 19-25 \mathrm{nt}$ in length. They regulate gene expression usually by targeting the $3^{\prime}$-UTR of their target mRNAs for translational repression, degradation, or both. ${ }^{1}$ According to the current researches, miRNAs involved in most of the biological processes in mammal cells, including proliferation, differentiation, migration, apoptosis, malignant transformation, metabolism, and so on. It is estimated that approximately $60 \%$ genes are regulated by miRNAs. ${ }^{2}$

miRNAs were first associated with cancer in $2002,{ }^{3}$ and the expressions of miRNA$15 \mathrm{a}$ as well as miRNA-16-1 were found to be decreased in B-cell chronic lymphocytic leukemia, which implied their potential role in cancer diagnosis and treatment. During the following decade, the abnormal expression pattern of many miRNAs was proved, ${ }^{2,4}$ and gradually, miRNAs were accepted as cancer suppressors or promoters.

MicroRNA-34a (miR-34a) expression was first evaluated in malignant cholangiocytes cells in $2006 .^{5}$ In the next year, miR-34a was found to be directly transactivated by $\mathrm{p} 53$, and its responsive genes were highly enriched for those that regulate cell cycle progression, apoptosis, DNA repair, and angiogenesis. ${ }^{6-9}$ In colon cancer cells, miR-34a suppressed cell proliferation and induced senescence-like phenotypes by downregulating the E2F pathway in vitro, and its decreased expression was also detected in cancer patients. ${ }^{10}$ Some other researches showed the similar results, ${ }^{11-15}$ so miR-34a was considered a tumor-suppressive miRNA. 
In 2009, a clinical research found that miR-34a was downregulated in non-small cell lung cancer (NSCLC) tissue, and its decreased expression was correlated with a high probability of relapse $(P=0.04)$, which was the first time that miR-34a was identified as a prognostic marker for cancer patients. ${ }^{16} \mathrm{Up}$ to now, overexpressed miR-34a has been reported to be related to good survival in NSCLC, ${ }^{17}$ pancreatic ductal adenocarcinoma, ${ }^{18}$ sinonasal squamous cell carcinoma, ${ }^{19}$ Ewing's sarcoma ${ }^{20}$ mantle cell lymphoma, ${ }^{21}$ mucosa-associated lymphoid tissue lymphoma and diffuse large B-cell lymphoma, ${ }^{22}$ as well as glioma. ${ }^{23}$ However, there were still insignificant or even opposite results. ${ }^{24-30}$ Therefore, it is necessary to conduct a systematic review and meta-analysis to get a better understanding of the prognostic effect of miR-34a on cancer patients.

In this research, we collected global literatures on the prognostic effect of miR-34a on cancer patients and assessed the value of miR-34a as a biomarker for good survival.

\section{Materials and methods}

The current analysis was conducted following the guidelines of Preferred Reporting Items for Systematic Reviews and Meta-Analyses (PRISMA) 2009 Checklist (http://www. prisma-statement.org/statement.htm) and Meta-analysis of Observational Studies in Epidemiology group (MOOSE; Table S1). ${ }^{31}$

\section{Identification of eligible studies}

We carefully searched the online PubMed (http://www.ncbi. nlm.nih.gov/pubmed) and Embase (http://www.embase.com/ home) from January 1st 2002 to April 19th 2014 to collect related literatures. The searching details were "miR-34a (all fields) and ('neoplasms' [MeSH terms] or 'neoplasms' [all fields] or 'cancer' [all fields])", "miR-34a (all fields) and ('carcinoma' [MeSH terms] or 'carcinoma' [all fields])", "miR-34a (all fields) and ('tumor' [all fields] or 'neoplasms' [MeSH terms] or 'neoplasms' [all fields] or 'tumor' [all fields])", “miR-34a (all fields) and ("neoplasms' [MeSH terms] or 'neoplasms' [all fields] or 'neoplasm' [all fields])", in PubMed and '“mir 34a'/exp or 'mir 34a' and ('cancer'/exp or cancer)", "'mir 34a'/exp or 'mir 34a' and ('carcinoma'/exp or carcinoma)", “'mir 34a'/exp or 'mir 34a' and ('tumor'/exp or tumor)", “'mir 34a'/exp or 'mir 34a' and ('neoplasm'/exp or neoplasm)" in Embase. We set no advanced limitations when searching both the databases. All the searching results were checked by going through the titles and abstracts, and the duplications were removed directly.

The eligible studies were collected using our previous methods..$^{32}$ Information of the eligible reports, such as titles, abstracts, and full texts, was independently and carefully identified by three reviewers (Jian Wang, Guorong Dan, and Feng Ye), and these extracted articles were checked for a second time by two reviewers (Yu Ding and Jin Cheng). All disagreements were discussed by the aforementioned reviewers or in consultation with two senior reviewers (Fahuan Yuan and Zhongmin Zou).

\section{Quality assessment}

All the included studies for survival analysis were evaluated according to the critical review checklist of the Dutch Cochrane Centre proposed by MOOSE. ${ }^{31}$ As described in detail previously, ${ }^{32}$ studies were excluded if they did not mention all the seven points.

\section{Data extraction and conversion}

Data were extracted in standardized data collection form. With regard to the researches on the prognostic effect of miR-34a on cancer survival, the extracted data included the following details: 1) publication information as mentioned earlier; 2) patient characteristics, including sample size, type of disease, stage of disease, histological type, and follow-up time; 3) miR-34a measurement and cutoff value; and 4) hazard ratios (HRs) of elevated miR-34a for overall survival (OS), disease-free survival, disease-specific survival (DSS), event-free survival (EFS), progress-free survival, recurrencefree survival, as well as their $95 \%$ confidence intervals (CIs) and $P$-values. If available, the HRs with their $95 \%$ CIs and $P$-values were directly collected from the original articles or Email correspondence with the corresponding authors. If not, the HRs and their 95\% CIs were calculated using the data of observed deaths/cancer recurrences, the data of samples in each group, or the data provided by the authors. If only Kaplan-Meier curves were available, essential data were extracted from the graphical survival plots and the HRs were estimated. All the aforementioned calculations were based on the methods provided by Parmar et $\mathrm{al}^{33}$ and Tierney et al ${ }^{13}$ which we used in our previous meta-analysis. ${ }^{32}$

\section{Statistical analysis}

The test of heterogeneity of combined HRs was carried out using Cochran's $Q$ test and Higgins $I$-squared statistic. The factors contributing to the heterogeneities were analyzed by subgroup analysis or sensitive analysis according to our previous methods. ${ }^{32}$ Publication bias was evaluated using the funnel plot with the Egger's bias indicator test. ${ }^{35}$ The other details were described in our previous analysis. ${ }^{32}$ All analyses were performed using "STATA: Data Analysis and Statistical Software" V11. 


\section{Results}

\section{Study characteristics and systematic review}

We collected 1,005 records from PubMed and 2,986 from Embase in the primary research with different keywords and excluded 3,015 duplicates from the initial records. After screening the titles, abstracts, publication types, and full texts of the remaining 1,005 records, 990 records were excluded according to the listed criteria. Then, the references of the remaining 15 qualified records were manually checked, and there was no additional record found in the cross-references. So, we got the 15 records included in our analysis. ${ }^{16-30}$ Figure 1 showed the flow diagram of candidate selection records in our study.

All the 15 studies were retrospective cohort researches in design. The collected 2,597 patients were from Austria, Belgium, Brazil, People's Republic of China, Finland, France, Germany, Greece, Italy, Japan, Lithuania, Poland, Romania, Slovakia, Spain, Sweden, UK, US, and Yugoslavia. The sample sizes ranged from 24 to 884 with an average sample size of 173 per study. The patients were diagnosed with a variety of cancers, including acute myeloid leukemia with complex karyotype, glioma, leukemic mantle cell lymphoma, resectable pancreatic ductal adenocarcinoma, glioblastoma, NSCLC, breast cancer, sinonasal squamous cell carcinoma, Ewing's sarcoma of bone, gastric cancer, esophageal adenocarcinoma, ovarian cancer, gastric mucosaassociated lymphoid tissue lymphoma, and diffuse large
B-cell lymphoma. Of all the 15 studies, four studies focused on digestive system tumor $(\mathrm{n}=351),{ }^{18,22,28,29}$ three studies on NSCLC $(n=742),{ }^{16,17,24}$ two studies on glioma $(n=302),{ }^{23,26}$ and two studies on lymphoma $(\mathrm{n}=152){ }^{21,22}$ Ten of the studies provided the stage of cancer patients, while the remaining five records did not. Thirteen of the 15 studies detected the expression of miR-34a in cancer tissue (six frozen and seven formalin fixed and paraffin embedded), one study detected in blood sample, ${ }^{29}$ and one study mentioned nothing about the concerning information. ${ }^{26} \mathrm{Six}$ of the included studies used median value as cutoff value, ${ }^{18,20,24,25,27,29}$ three studies used exact value of miRNA expression, ${ }^{17,19,23}$ two studies used values selected by Maxstat software, ${ }^{16,21}$ one study used the first quartile of the Automated Quantitative Analysis score distribution, ${ }^{26}$ and three did not mention the concerning information. For criteria of survival assessment, ten of the included studies used OS, two used recurrence-free survival, two used progress-free survival, two used disease-free survival, one used DSS, one used cancer-specific survival, and one used EFS. The HRs were acquired by direct collection of reported data in six records, calculation based on the shown data in three records, evaluation from the survival curve in five researches, and author's Email for one research. The follow-up time ranged from 23 to 150 months and reached 5 years in ten studies. The main information of the 15 studies was summarized in Table 1.

To measure the expression of miR-34a, 12 of the 15 studies used qRT-PCR, two used in situ hybridization, ${ }^{22,28}$ and

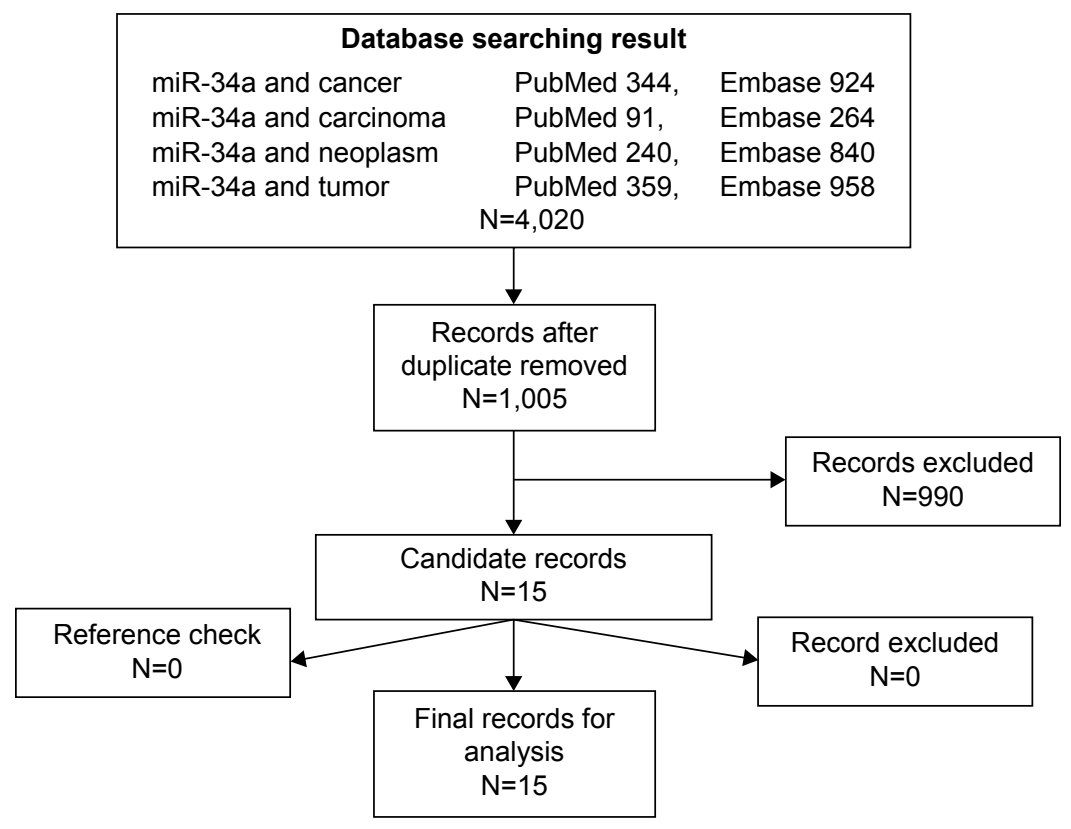

Figure I The flowchart showed the selection of studies for meta-analysis.

Abbreviation: miR-34a, microRNA-34a. 


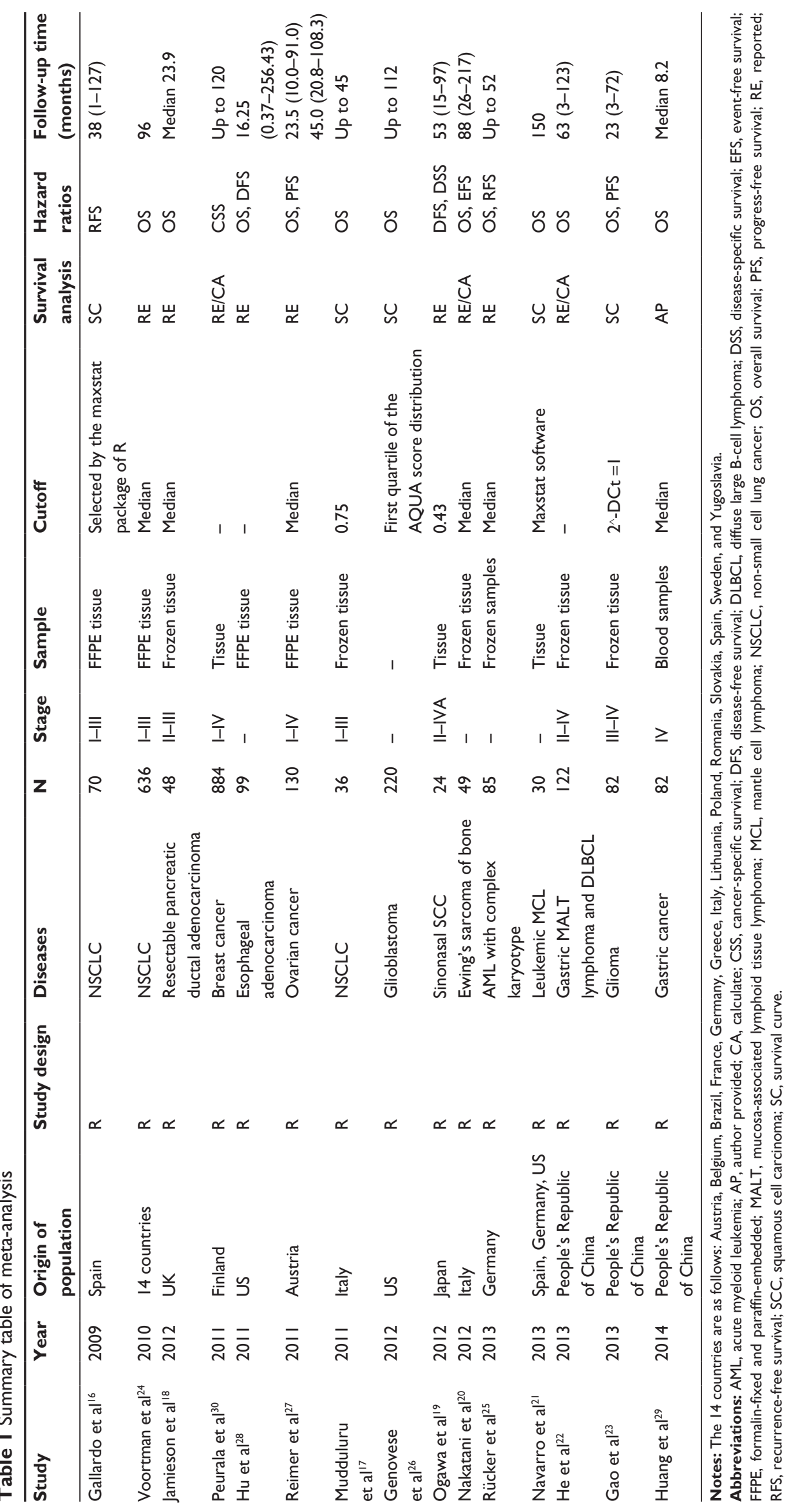


one used microarray. ${ }^{30}$ In the qRT-PCR studies, three used $\mathrm{U} 6 \mathrm{~B}$ as their internal reference, ${ }^{17,23,25}$ three used U6, ${ }^{18,20,22}$ and miR-16, ${ }^{29}$ miR-191, ${ }^{16} \mathrm{U} 44,{ }^{19} \mathrm{U} 48,{ }^{21} \mathrm{U} 66,{ }^{24}$ or $\mathrm{TBP}^{27}$ was used in one study separately. With regard to risk evaluation methods, five researches used Kaplan-Meier curves and the rest ten researches used Multiple Cox proportional hazard model. Seven of the 15 researches collected their cancer samples after a period of treatment, seven researches declared that their samples were collected before any clinical treatment, and the rest one research neglected the related information. All the assay details were summarized in Table 2.

\section{Meta-analysis results}

HRs could only be retrieved from the unique study, such as DSS (HR $=0,95 \% \mathrm{CI}$ : 0 ) in sinonasal squamous cell carcinoma, cancer-specific survival in breast cancer (5-year HR $=0.88,0.65-1.88, P=0.573 ; 10$-year HR $=0.67,95 \%$ CI: $0.50-0.89, P=0.073$ ), and $\mathrm{EFS}$ (HR $=0.491,95 \% \mathrm{CI}$ : $0.40-0.60, P=0.0004)$. If more than two researches fall into one category, the HRs were pooled. For studies evaluating OS of patients, a pooled HR and its $95 \%$ CI were calculated with a random model because of the high heterogeneity between studies ( $P=0.000, I^{2}=79.4 \%$ ). The result showed that higher expression of miR-34a may predict good OS, and the pooled HR was 0.76 (95\% CI: 0.55-1.06); however, the effect did not reach the level of statistical significance $(P=0.105)$ (Figure 2).

Furthermore, subgroup analysis was carried out based on the types of cancers, such as digestive system cancer, lymphoma, and sarcoma. First, as an obvious heterogeneity ( $P=0.002$ and $\left.I^{2}=84.0 \%\right)$ existed in those researches on OS of digestive system cancer patients, a random model was used to pool the HRs. The combined HR, 0.51 (95\% CI: $0.20-1.26, P=0.145$ ) (Figure 3A), indicated that overexpressed miR-34a would potently predict good OS for patients with digestive system cancer. Two researches focused on the prognostic effect of miR-34a on the OS of lymphoma patients and sarcoma patients separately, and meta-analysis was not conducted.

Seeing that the expression of miR-34a in some researches was detected in cancer samples collected before treatment, and some was after treatment, HRs was also pooled according to these two conditions. A random model was used to pool the HRs of researches with untreated patients (low heterogeneity, $P=0.060$ and $P=59.5 \%$ ), and the combined HR was 0.63 (95\% CI: 0.38-1.06, $P=0.084$ ) (Figure 4A). Because an obvious heterogeneity $\left(P=0\right.$ and $\left.I^{2}=84.4 \%\right)$ existed among six researches with treated patients, a random model was used to pool the HRs. The combined HR, 0.79 (95\% CI: $0.51-1.21, P=0.271$ ) (Figure 4B), was comparable with the pooled HR of untreated patients, suggesting that the treatment did not significantly influence the predictive effect of miR-34a.

\section{Possible sources of the heterogeneity}

Obvious heterogeneity of subjects was found in all the four analysis groups (OS for all, $P=0$ and $P=74.9 \%$; OS for digestive system cancer, $P=0.004$ and $I^{2}=77.7 \%$; OS for treated patients, $P=0$ and $P=84.4 \%$; and OS for untreated patients, $P=0.060$ and $P^{2}=59.5 \%$ ). The most possible sources of the heterogeneity were also analyzed by different methods.

On the one hand, since the heterogeneity of OS analysis group was obvious, we divided the 11 studies into three cancer type-specific analysis groups (four studies on digestive system cancer, two studies for lymphoma, and two studies on sarcoma). The heterogeneity was still obvious in digestive system cancer and sarcoma groups, so the cancer type could not solely explain the heterogeneity in OS analysis group. On the other hand, a meta-regression analysis was conducted to evaluate the potential factors responsible for the obvious heterogeneity. As a result, the publication year $(P=0.617)$, cutoff values $(P=0.651)$, patient's origin $(P=0.914)$, risk evaluation method $(P=0.957)$, follow-up time $(P=0.751)$, and cancer type $(P=0.112)$ contributed little to the heterogeneity, but the sample size ( $P=0.023)$ was the main source of heterogeneity.

Because meta-regression analysis is not proper to seek the sources of heterogeneity for analysis group with less than ten studies, sensitivity analysis was performed instead. In the OS analysis group for untreated patients, heterogeneity was significant $\left(P=0.060\right.$ and $\left.P^{2}=59.5 \%\right)$. When Nakatani's study was removed from analysis, the heterogeneity became insignificant ( $P=0.704$ and $P=0 \%$ ). Using the same method, we found that Jamieson's studies were responsible for the heterogeneity in the digestive system cancer analysis group, and Jamieson's as well as He's studies contributed most to the heterogeneity in the treated patients group.

\section{Publication bias}

The publication bias of included studies was evaluated by funnel plots and Egger's tests. As shown in Figure 3B, the funnel plots were almost symmetric. In OS meta-analysis, the $P$-values of Egger's regression intercepts were 0.175 . Hence, there was no evidence for significant publication bias in our meta-analysis. 


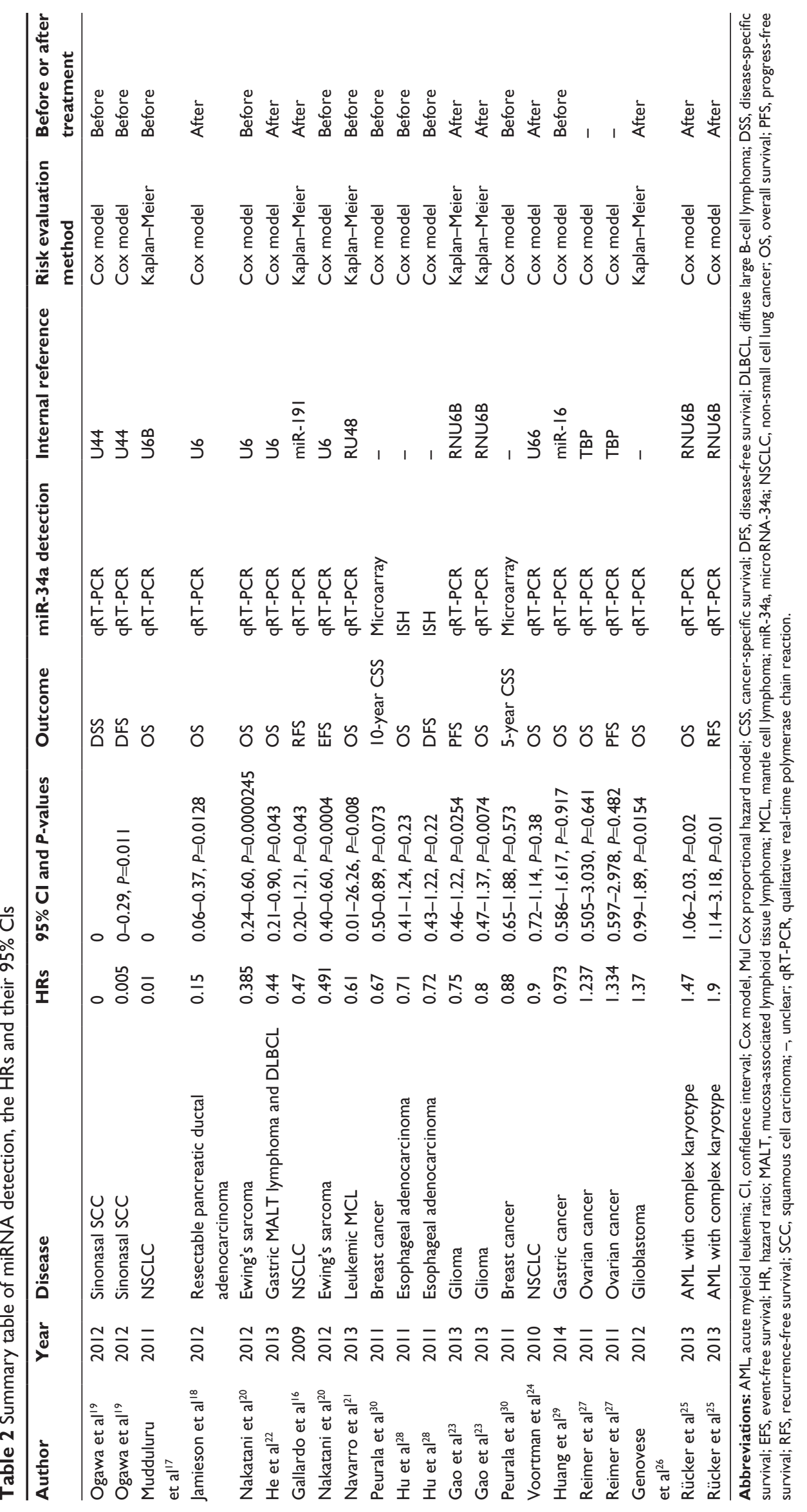




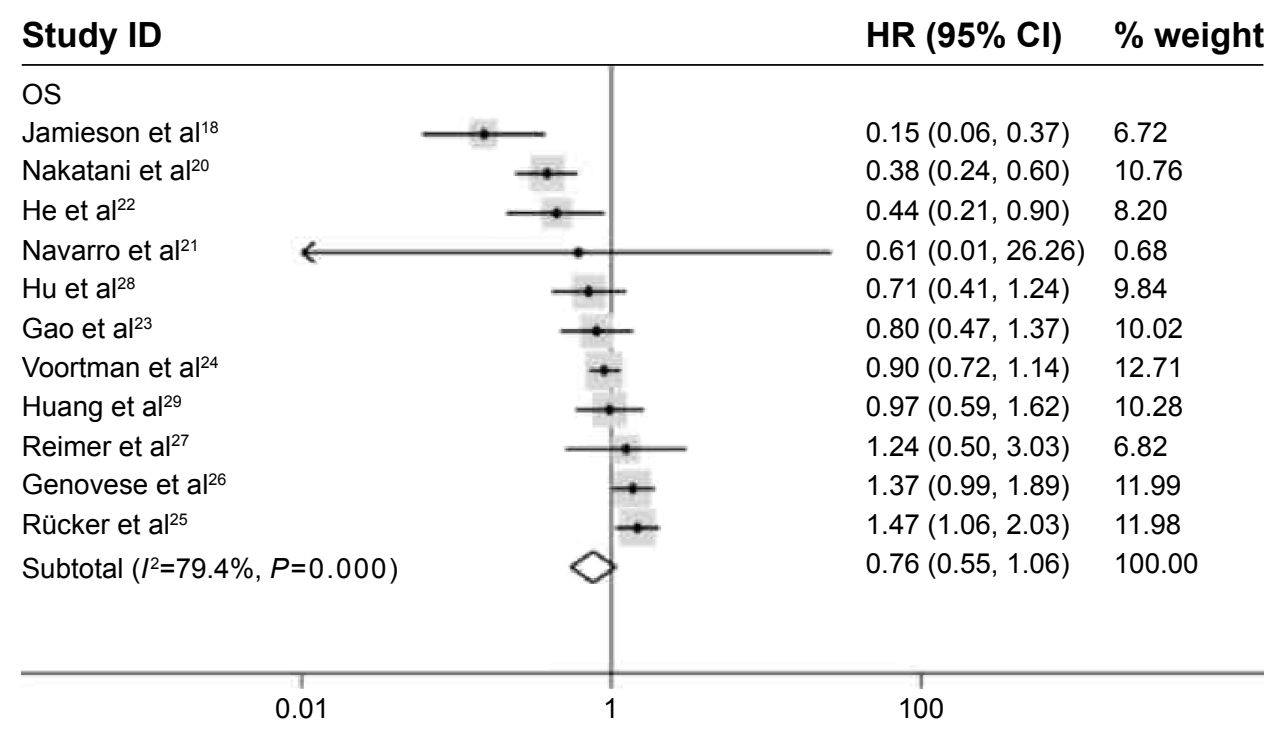

Figure 2 Forrest plots of studies evaluating hazard ratios of high miR-34a expression. The random effects analysis model showed the pooled HR for overall survival is 0.76 with $95 \% \mathrm{Cl}: 0.55-1.06$, and $P$-value is 0.105 .

Note: Weights are from random effects analysis.

Abbreviations: $\mathrm{Cl}$, confidence interval; miR-34a, microRNA-34a; OS, overall survival; HR, hazard ratio; ID, identification.

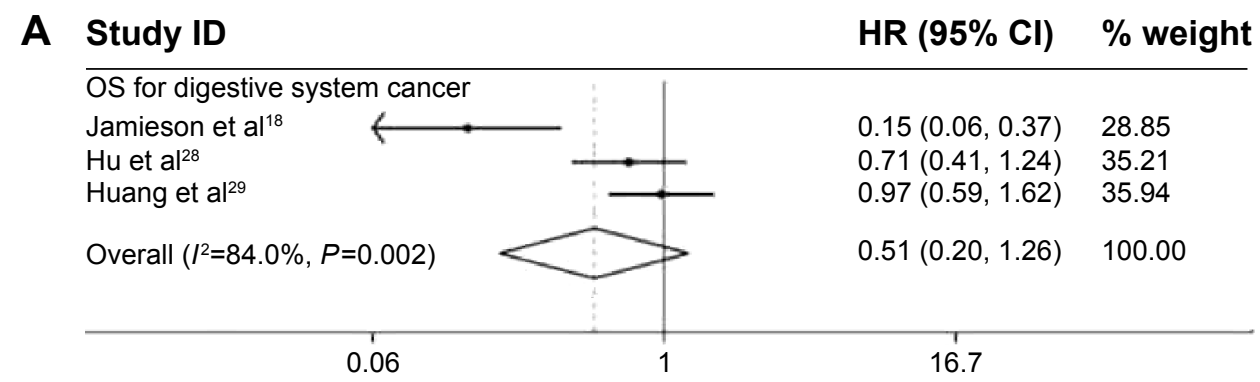

B Begg's funnel plot with pseudo 95\% confidence limits

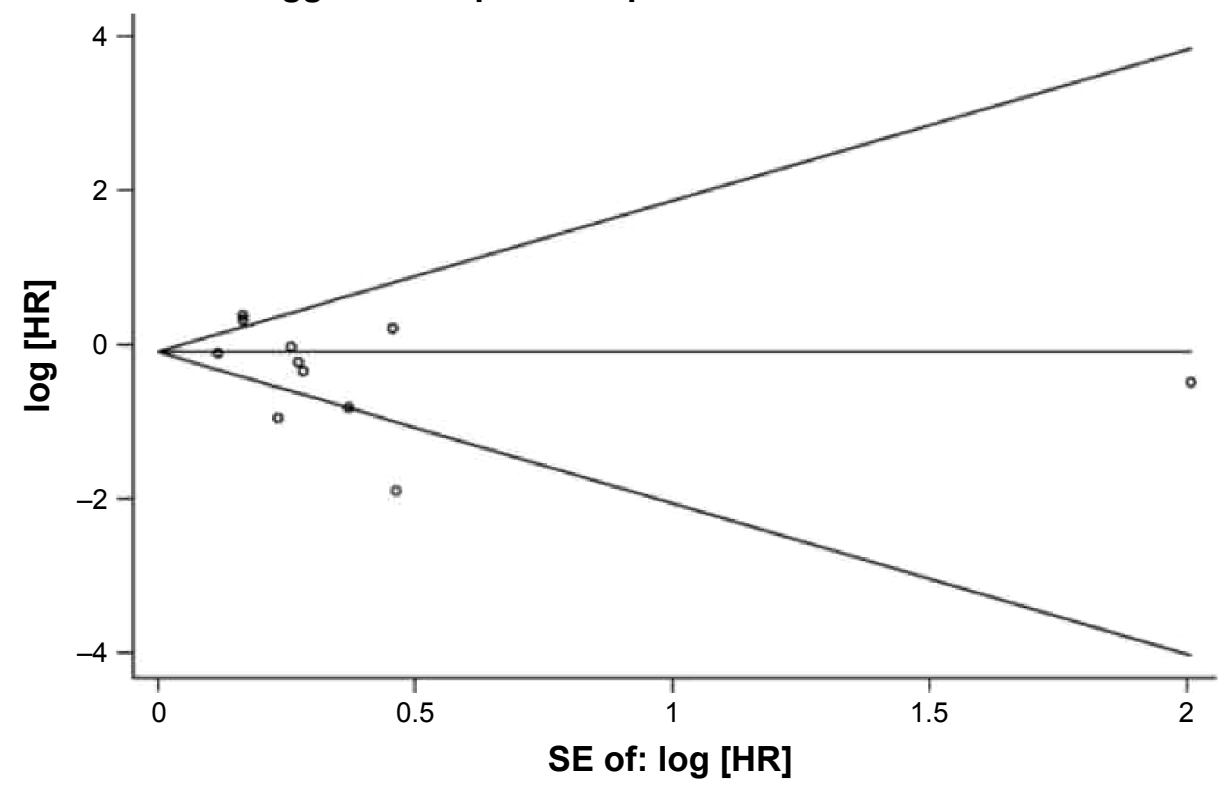

Figure 3 Forrest plots of studies evaluating hazard ratios of high miR-34a expression and Funnel plots of studies included in meta-analysis.

Notes: (A) The random effects analysis model showed the pooled HR for digestive system cancer patients overall survival is 0.5 I with $95 \%$ Cl: $0.20-1.26$, and $P$-value is 0.146 . (B) Funnel plots of studies assessed the prognostic effect of miR-34a on overall survival. Weights are from random effects analysis.

Abbreviations: $\mathrm{Cl}$, confidence interval; miR-34a, microRNA-34a; OS, overall survival; HR, hazard ratio; SE, standard error; ID, identification. 


\section{A Study ID}

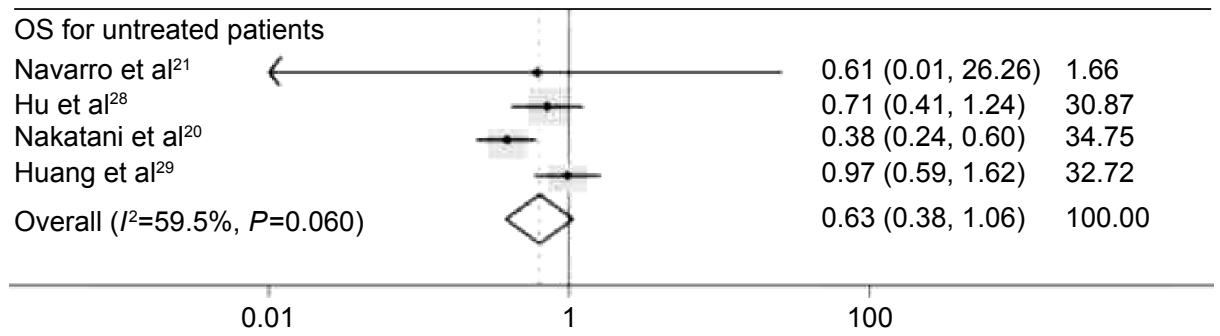

\section{$\mathrm{HR}(95 \% \mathrm{Cl}) \quad \%$ weight}

$0.61(0.01,26.26) \quad 1.66$

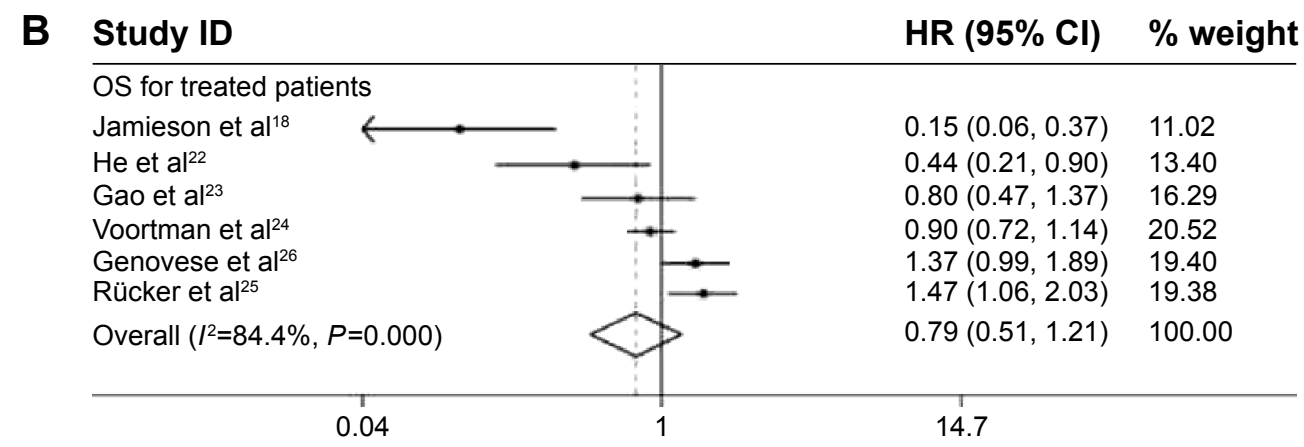

Figure 4 Subgroup analysis results.

Notes: (A) The random effects analysis model showed the pooled HR for overall survival of untreated patients is 0.63 with $95 \% \mathrm{Cl}: 0.38-1.06$, and $P$-value is 0.084 . (B) The random effects analysis model showed the pooled $\mathrm{HR}$ for overall survival of treated patients is 0.79 with $95 \% \mathrm{Cl}$ : $0.5 \mathrm{I}-\mathrm{I} .2 \mathrm{I}$, and $P$-value is $0.27 \mathrm{I}$. Weights are from random effects analysis.

Abbreviations: $\mathrm{Cl}$, confidence interval; OS, overall survival; $\mathrm{HR}$, hazard ratio; ID, identification.

\section{Discussion}

miR-34 family includes three members-miR-34a, miR-34b, and miR-34c. In human, miR-34a is located at $1 \mathrm{p} 36$ locus of chromosome 1, and miR-34b and miR-34c are located at 11q23 locus of chromosome 11. All the three members are p53-dependent and share the same seed sequence as well as similar functions, but their target genes are different. ${ }^{36}$ As a downstream element of p53 pathway, miR-34a is always known as a tumor-suppressive miRNA for its repression effect on cell cycle, cell invasion, cell migration, cancer stem cell, and so on. ${ }^{37}$ miR-34a plays an important role in DNA damage response. Ectopic expression of miR-34a induces cell cycle arrest by downregulating target genes, and as a result, inhibits damaged DNA replication. ${ }^{6}, 10$ The expression of miR-34a decreases frequently in some p53 mutant cancer cells, such as U251 and chronic lymphocytic leukemia cells. In these cells, some miR-34a target genes, which are related to cell cycle, tumor invasion, and migration, are upregulated, ${ }^{39,40}$ and restoration of functional miR-34a enhances the chemotherapy susceptibility and inhibits tumor cell growth. Some of the direct downstream targets of miR-34a, such as Bcl-2, Notch, and HMGA2 (high-mobility group AT-hook 2), are all responsible for self-renewal of the cell, so miR-34a may suppress the self-renewal of cancer stem cells. ${ }^{36,41}$
In animal tumor models, the therapeutic activity of miR-34a was also evaluated in NSCLC, prostate cancer, melanoma, pancreatic cancer, and lymphoma, and the miR-34a treated animal showed a significant tumor growth inhibition. ${ }^{37}$ These experiment results were consistent to our research.

In the current analysis, we got four pooled HRs from 15 researches on 2,597 patients with 13 different types of cancer from 19 countries. The main conclusions can be summarized as follows: 1) miR-34a did predict good overall survival for cancer patients with a pooled HR $=0.76$ (95\% CI: 0.55-1.06), though the $P$-value was not satisfying enough $(P=0.105)$; $2)$ the result seemly suggested that miR-34a was an ideal biomarker for good outcome in digestive system cancer patients (HR $=0.50,95 \%$ CI: $0.25-0.99, P=0.048) ; 3$ ) the predictive role of miR-34a in cancer tissue collected from patients before ( $\mathrm{HR}=0.63,95 \% \mathrm{CI}$ : $0.38-1.06, P=0.084)$ and after $(\mathrm{HR}=0.79,95 \% \mathrm{CI}: 0.51-1.21, P=0.271)$ treatment, and they were not obviously different. To our knowledge, this was the first meta-analysis on the prognostic effect of miR-34a on cancer patients.

Though the prognostic effect role of miR-34a on cancer patients was proved by experimental results and statistically identified by clinical researches and this meta-analysis, 
current conclusions should be cautiously appreciated. First, all the four pooled HRs were of statistical insignificance, suggesting the predictive effect of miR-34a was limited. Second, several HRs were calculated based on the data collected from the survival curves, which would bring errors, although small. Third, HRs were pooled from different articles with different cutoff values due to methods limitations while a general baseline of miR-34a expression level could not be set up. In addition, the HR for OS in Mudduluru's study was 0.01 with $95 \%$ CI of 0 , which made the direct pooling of this HR with the others using STATA impossible, so the HRs were pooled without Mudduluru's study. Fourth, only researches published in English were included in this analysis, which could lead to the miss of applicable studies in non-English publication.

Because six of the eight analysis groups were with heterogeneity, the HRs were pooled with a random effect model, and the possible resource of heterogeneity was also explored by subgroup analysis, meta-regression, and sensitive analysis. Subgroup analysis based on cancer types as well as the meta-regression result suggested that the cancer type might not be responsible for the heterogeneity. The meta-regression results showed that the sample size of each study was responsible for the obvious heterogeneity in OS analysis group, and the sensitive analysis results figured out responsible studies of heterogeneity in the analysis groups of treated patients, untreated patients, and digestive system cancer patients. Publication bias was not found in the OS analysis group; however, the amount of researches was not large enough to ensure the current conclusion.

Nowadays, miRNAs have been widely considered as oncogene or cancer suppressor, but several concerns should be stressed. First, a set of miRNAs would be more proper to predict the outcome of one type of cancer. There are already studies focused on this issue, ${ }^{42,43}$ and present analysis proposes miR-34a as a candidate miRNA for future study. Second, most of the studies detected the expression of miR-34a in cancer tissue, and this brings many problems. Due to the heterogeneity of cancer, the collected tissue might not precisely reflect the status of cancer, and lack of standard methods of collecting the tissue, isolating the RNA, detecting the expression of miRNA, and even the internal references would obstruct the clinical research of miRNA in cancer. Third, circulating miRNAs would be an ideal choice for their convenient sampling in future clinical cancer research. Circulating miRNA may not only play diagnostic and prognostic role in cancer patients but also can be applied in cancer screening by regularly monitoring miRNA profiles.

\section{Conclusion}

Our meta-analysis collected studies on the relationship of miR-34a expression and cancer patient survival found that the overexpression of miR-34a potently predict good survival of patients with digestive system cancer. Because of the limitation of meta-analysis results when applied to observational or retrospective studies that could hardly be compared, one should be cautious in interpreting the current conclusion. Further clinical researches are needed to testify the association between miR-34a and cancer prognosis as well as the efficiency of therapies.

\section{Acknowledgment}

This research was supported by the Major Military Medical S \& T Project of PLA for the "Twelfth Five-year Plan" (AWS11C004) and National Natural Science Foundation of China (grant number 81302862).

\section{Disclosure}

The authors declare no conflict of interests in this work.

\section{References}

1. Ambros V. The functions of animal microRNAs. Nature. 2004; 431(7006):350-355.

2. Esteller M. Non-coding RNAs in human disease. Nat Rev Genet. 2011;12(12):861-874.

3. Guo H, Ingolia NT, Weissman JS, Bartel DP. Mammalian microRNAs predominantly act to decrease target mRNA levels. Nature. 2010;466(7308):835-840.

4. Calin GA, Croce CM. MicroRNA signatures in human cancers. Nat Rev Cancer. 2006;6(11):857-866.

5. Meng $\mathrm{F}$, Henson R, Lang M, et al. Involvement of human micro-RNA in growth and response to chemotherapy in human cholangiocarcinoma cell lines. Gastroenterology. 2006;130(7):2113-2129.

6. Chang TC, Wentzel EA, Kent OA, et al. Transactivation of miR-34a by p53 broadly influences gene expression and promotes apoptosis. Mol Cell. 2007;26(5):745-752.

7. He L, He X, Lim LP, et al. A microRNA component of the $\mathrm{p} 53$ tumour suppressor network. Nature. 2007;447(7148):1130-1134.

8. He X, He L, Hannon GJ. The guardian's little helper: microRNAs in the p53 tumor suppressor network. Cancer Res. 2007;67(23): 11099-11101.

9. Raver-Shapira N, Marciano E, Meiri E, et al. Transcriptional activation of miR-34a contributes to p53-mediated apoptosis. Mol Cell. 2007;26(5):731-743.

10. Tazawa H, Tsuchiya N, Izumiya $M$, Nakagama $H$. Tumor-suppressive miR-34a induces senescence-like growth arrest through modulation of the E2F pathway in human colon cancer cells. Proc Natl Acad Sci U SA. 2007;104(39):15472-15477.

11. Welch C, Chen Y, Stallings RL. MicroRNA-34a functions as a potential tumor suppressor by inducing apoptosis in neuroblastoma cells Oncogene. 2007;26(34):5017-5022.

12. Zanette DL, Rivadavia F, Molfetta GA, et al. miRNA expression profiles in chronic lymphocytic and acute lymphocytic leukemia. Braz J Med Biol Res. 2007;40(11):1435-1440.

13. Cole KA, Attiyeh EF, Mosse YP, et al. A functional screen identifies miR-34a as a candidate neuroblastoma tumor suppressor gene. Mol Cancer Res. 2008;6(5):735-742. 
14. Kumamoto K, Spillare EA, Fujita K, et al. Nutlin-3a activates p53 to both down-regulate inhibitor of growth 2 and up-regulate miR-34a, miR-34b, and miR-34c expression, and induce senescence. Cancer Res. 2008;68(9):3193-3203.

15. Rokhlin OW, Scheinker VS, Taghiyev AF, Bumcrot D, Glover RA, Cohen MB. MicroRNA-34 mediates AR-dependent p53-induced apoptosis in prostate cancer. Cancer Biol Ther. 2008;7(8):1288-1296.

16. Gallardo E, Navarro A, Viñolas N, et al. miR-34a as a prognostic marker of relapse in surgically resected non-small-cell lung cancer. Carcinogenesis. 2009;30(11):1903-1909.

17. Mudduluru G, Ceppi P, Kumarswamy R, Scagliotti GV, Papotti M, Allgayer H. Regulation of Axl receptor tyrosine kinase expression by miR-34a and miR-199a/b in solid cancer. Oncogene. 2011;30(25):2888-2899.

18. Jamieson NB, Morran DC, Morton JP, et al. MicroRNA molecular profiles associated with diagnosis, clinicopathologic criteria, and overall survival in patients with resectable pancreatic ductal adenocarcinoma. Clin Cancer Res. 2012;18(2):534-545.

19. Ogawa T, Saiki Y, Shiga K, et al. miR-34a is downregulated in cisdiamminedichloroplatinum treated sinonasal squamous cell carcinoma patients with poor prognosis. Cancer Sci. 2012;103(9):1737-1743.

20. Nakatani F, Ferracin M, Manara MC, et al. miR-34a predicts survival of Ewing's sarcoma patients and directly influences cell chemo-sensitivity and malignancy. J Pathol. 2012;226(5):796-805.

21. Navarro A, Clot G, Prieto M, et al. microRNA expression profiles identify subtypes of mantle cell lymphoma with different clinicobiological characteristics. Clin Cancer Res. 2013;19(12):3121-3129.

22. He M, Gao L, Zhang S, et al. Prognostic significance of miR-34a and its target proteins of FOXP1, p53, and BCL2 in gastric MALT lymphoma and DLBCL. Gastric Cancer. 2014;17(3):431-441.

23. Gao H, Zhao H, Xiang W. Expression level of human miR-34a correlates with glioma grade and prognosis. J Neurooncol. 2013; 113(2):221-228.

24. Voortman J, Goto A, Mendiboure J, et al. MicroRNA expression and clinical outcomes in patients treated with adjuvant chemotherapy after complete resection of non-small cell lung carcinoma. Cancer Res. 2010;70(21):8288-8298.

25. Rücker FG, Russ AC, Cocciardi S, et al. Altered miRNA and gene expression in acute myeloid leukemia with complex karyotype identify networks of prognostic relevance. Leukemia. 2013;27(2):353-361.

26. Genovese G, Ergun A, Shukla SA, et al. microRNA regulatory network inference identifies miR-34a as a novel regulator of TGF-beta signaling in glioblastoma. Cancer Discov. 2012;2(8):736-749.

27. Reimer D, Hubalek M, Kiefel H, et al. Regulation of transcription factor E2F3a and its clinical relevance in ovarian cancer. Oncogene. 2011;30(38):4038-4049.
28. Hu Y, Correa AM, Hoque A, et al. Prognostic significance of differentially expressed miRNAs in esophageal cancer. Int J Cancer. 2011; 128(1):132-143.

29. Huang D, Wang H, Liu R, et al. miRNA27a is a biomarker for predicting chemosensitivity and prognosis in metastatic or recurrent gastric cancer. J Cell Biochem. 2014;115(3):549-556.

30. Peurala H, Greco D, Heikkinen T, et al. miR-34a expression has an effect for lower risk of metastasis and associates with expression patterns predicting clinical outcome in breast cancer. PLoS One. 2011; 6(11):e26122.

31. Stroup DF, Berlin JA, Morton SC, et al. Meta-analysis of observational studies in epidemiology: a proposal for reporting. Meta-analysis of Observational Studies in Epidemiology (MOOSE) Group. JAMA. 2000;283(15):2008-2012.

32. Wang J, Zhao J, Shi M, et al. Elevated expression of miR-210 predicts poor survival of cancer patients: a systematic review and meta-analysis. PLoS One. 2014;9(2):e89223.

33. Parmar MK, Torri V, Stewart L. Extracting summary statistics to perform meta-analyses of the published literature for survival endpoints. Stat Med. 1998;17(24):2815-2834.

34. Tierney JF, Stewart LA, Ghersi D, Burdett S, Sydes MR. Practical methods for incorporating summary time-to-event data into meta-analysis. Trials. 2007;8:16.

35. Egger M, Davey Smith G, Schneider M, Minder C. Bias in meta-analysis detected by a simple, graphical test. BMJ. 1997;315(7109):629-634.

36. Hermeking H. The miR-34 family in cancer and apoptosis. Cell Death Differ. 2010;17(2):193-199.

37. Bader AG. miR-34 - a microRNA replacement therapy is headed to the clinic. Front Genet. 2012;3:120.

38. Ichimura A, Ruike Y, Terasawa K, Shimizu K, Tsujimoto G. MicroRNA$34 \mathrm{a}$ inhibits cell proliferation by repressing mitogen-activated protein kinase kinase 1 during megakaryocytic differentiation of K562 cells. Mol Pharmacol. 2010;77(6):1016-1024.

39. Zenz T, Mohr J, Eldering E, et al. miR-34a as part of the resistance network in chronic lymphocytic leukemia. Blood. 2009;113(16):3801-3808.

40. Luan S, Sun L, Huang F. MicroRNA-34a: a novel tumor suppressor in p53-mutant glioma cell line U251. Arch Med Res. 2010;41(2):67-74.

41. Ji Q, Hao X, Meng Y, et al. Restoration of tumor suppressor miR-34 inhibits human p53-mutant gastric cancer tumor spheres. BMC Cancer. 2008;8:266

42. Vosa U, Vooder T, Kolde R, Vilo J, Metspalu A, Annilo T. Metaanalysis of microRNA expression in lung cancer. Int $J$ Cancer. 2013;132(12):2884-2893

43. Cheng C, Wang Q, You W, Chen M, Xia J. MiRNAs as biomarkers of myocardial infarction: a meta-analysis. PLoS One. 2014;9(2):e88566. 


\section{Supplementary materials}

Table SI MOOSE checklist

Checklist items

Reporting of background should include

Problem definition

Hypothesis statement

Description of study outcome(s)

Type of exposure or intervention used

Type of study designs used

Study population

Reporting of search strategy should include

Qualifications of searchers (eg, librarians and investigators)

Search strategy, including time period included in the synthesis and keywords

Effort to include all available studies, including contact with authors

Databases and registries searched

Search software used, name and version, including special features used (eg, explosion)

Use of hand searching (eg, reference lists of obtained articles)

List of citations located and those excluded, including justification

Method of addressing articles published in languages other than English

Method of handling abstracts and unpublished studies

Description of any contact with authors

\section{Reporting of methods should include}

Description of relevance or appropriateness of studies assembled for assessing the hypothesis to be tested

Rationale for the selection and coding of data (eg, sound clinical principles or convenience)

Documentation of how data were classified and coded (eg, multiple raters, blinding, and interrater reliability)

Assessment of confounding (eg, comparability of cases and controls in studies where appropriate)

Assessment of study quality, including blinding of quality assessors; stratification or regression on possible

predictors of study results

Assessment of heterogeneity

Description of statistical methods (eg, complete description of fixed or random effects models, justification

of whether the chosen models account for predictors of study results, dose-response models, or

cumulative meta-analysis) in sufficient detail to be replicated

Provision of appropriate tables and graphics

Reporting of results should include

Graphic summarizing individual study estimates and overall estimate

Table giving descriptive information for each study included

Results of sensitivity testing (eg, subgroup analysis)

Indication of statistical uncertainty of findings

Reporting of discussion should include

Quantitative assessment of bias (eg, publication bias)

Justification for exclusion (eg, exclusion of non-English language citations)

Assessment of quality of included studies

Reporting of conclusions should include

Consideration of alternative explanations for observed results

Generalization of the conclusions (ie, appropriate for the data presented and within the domain of the

literature review)

Guidelines for future research

Disclosure of funding source
Related sections and indications

\author{
Background \\ Background \\ OS, DFS, PFS, RFS, DSS, EFS, CSS \\ Cancer \\ Systematic reviews and meta-analysis \\ Global
}

Stated in methods

Methods

We contact authors and searched

reference lists and citations

Methods

360 secured browser 6.2

Methods

Flow diagram in Figure I

Method

Method

Method

Method

Methods

Methods

Methods

Methods

Methods

Methods

Methods

Figures 2, 3

Tables I, 2

Figures 4

Discussion

Result

Discussion

Results and discussion

Discussion

Discussion

Discussion

Acknowledge

Abbreviations: OS, overall survival; DFS, disease-free survival; PFS, progress-free survival; RFS, recurrence-free survival; DSS, disease-specific survival; CSS, cancer-specific survival; MOOSE, Meta-analysis of Observational Studies in Epidemiology group.

OncoTargets and Therapy

\section{Publish your work in this journal}

OncoTargets and Therapy is an international, peer-reviewed, open access journal focusing on the pathological basis of all cancers, potential targets for therapy and treatment protocols employed to improve the management of cancer patients. The journal also focuses on the impact of management programs and new therapeutic agents and protocols on

\section{Dovepress}

patient perspectives such as quality of life, adherence and satisfaction. The manuscript management system is completely online and includes a very quick and fair peer-review system, which is all easy to use. Visit http://www.dovepress.com/testimonials.php to read real quotes from published authors. 